\title{
COMMUNICATIONS
}

\section{TREATMENT OF GROSS CORNEAL OPACIFICATION BY LAMELLAR AND ANNULAR LAMELLAR KERATOPLASTY*†}

\author{
BY \\ A. G. LEIGH \\ Institute of Ophthalmology, University of London
}

Perforating keratoplasty is found to be most successful in cases of small central corneal nebulae and keratoconus. In both these conditions the graft lies completely surrounded by healthy cornea. Where there is gross opacification extending widely throughout the thickness of the cornea and especially if degenerative changes are present, then a simple perforating graft rapidly becomes completely opaque and takes on the characteristics of the tissue into which it was placed. The presence of vascularization within the recipient cornea appears to accelerate this process.

Experience would show that a perforating graft, to survive as a clear structure, must be placed in a cornea of recognizable structure and that the opacities therein must not exceed two-thirds of the area and thickness of the cornea in contact with the graft.

The basic problem therefore is to establish within these grossly opaque, degenerative, and possibly vascularized corneae tissue which will maintain a perforating graft as an optically clear structure.

It is common knowledge that a lamellar graft placed in an opaque cornea frequently survives as clear corneal tissue and that in many cases some clarification of the surrounding cornea is seen. When such a lamellar graft has remained clear for a period of at least 6 months, then a smaller perforating keratoplasty may be successfully performed through its centre.

This is a valuable and effective method of dealing with a dense nonvascularized opacity no larger than $7 \times 8 \mathrm{~mm}$. and where the peripheral zone of the cornea is clear. An opacity of this size, it is true, could be successfully treated by a single, large perforating graft, but such a method has the following disadvantages:

(1) The operation is more difficult and hazardous than a 5-mm. graft and the attendant complications of anterior synechiae and peripheral anterior synechiae due to late reformation of the anterior chamber are more likely to occur.

(2) The assessment of the suitability of the peripheral cornea to support a clear graft is frequently difficult.

* Received for publication July 6, 1955.

+ The substance of this paper formed the basis of a communication to the Oxford Ophthalmological Congress in June, 1955. 
(3) Should a large perforating graft become opaque, the condition may then present a problem more difficult than that first seen.

In cases of gross corneal opacification associated with much superficial vascularization, I observed that after a lamellar keratoplasty had been performed the graft usually remained clear and free from either superficial or interstitial vascularization, but that a dense vascular network developed between the recipient cornea and the graft (Fig. 9). The blood supply to this plexus was derived from a few large vessels which passed from the vascularization at the limbus across the peripheral zone of the cornea, to reach the plexus by passing between the margin of the graft and the recipient cornea.

Before a perforating graft could be placed successfully through the lamellar grait, this vascularization had to be destroyed. Irradiation was ineffective for such large vessels and peritomy unsuccessful for the main vessels were

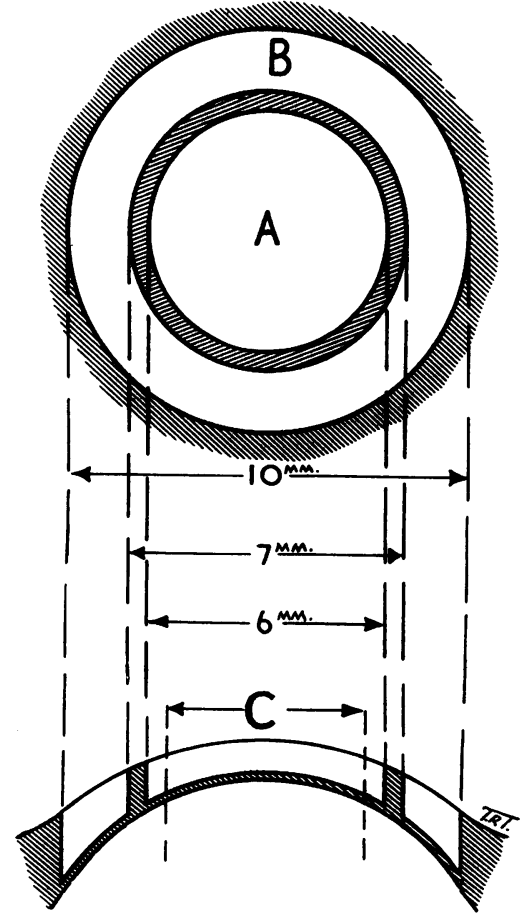

Fig. 1.-Principles of operative procedure.

(A) Initial 6-mm. lamellar graft.

(B) Annular graft.

(C) Final 5-mm. perforating graft. rapidly replaced by the development of some of the many adjacent small vessels.

It occurred to me that the vascularity might be overcome by placing an annular lamellar graft outside the central lamellar graft, a thin ridge of recipient cornea being left intervening between the inside edge of the annulus and the lamellar graft (Fig. 1). This operation proved to be a successful method of considerably reducing the vascularization beneath the lamel lar graft and establishing suitable tissue through which a perforating graft could be placed. Even in severe cases of peripheral vascularization, the vessels from the limbus, in attempting to reach the sub-lamellar plexus, rarely succeeded in crossing the annulus superficially but did so by passing to the under surface of the annulus, then over the ridge of the recipient cornea between the annulus and the lamellar graft, and thence between the lamellar graft and the recipient cornea. The few vessels which thus succeeded in establishing communication with the plexus were considerably attenuated as a result of traversing this devious route.

In addition to the effect upon vascularization, the annular graft, in many cases, remained clear and sometimes produced a small degree of clarification of the surrounding cornea and of the central lamellar graft. 
Observing this behaviour of the annular graft, I felt that a similar technique might be used effectively for the development within a grossly opaque cornea of tissue which could maintain a perforating graft as an optically clear structure.

\section{Technique}

The first stage of the operation is performed by placing a lamellar graft of $6 \mathrm{~mm}$. diameter and 0.4 to $0.5 \mathrm{~mm}$. thick, in a bed of exactly similar dimensions cut in the central area of the recipient cornea. The graft is centred by free-hand marking of the opaque cornea by means of a Graefe knife (Fig. 2). After staining with fluorescein, these marks form an excellent guide to the site of the trephine. I employ this method for all forms of corneal grafting. The cornea having been cut by the trephine to the required depth, the disc of tissue is now separated from the cornea by the use of a Paufique elevator which establishes accurately the plane of cleavage. Subsequent dissection and separation of the disc is performed by a Desmarres knife. The donor cornea is cut in an exactly similar fashion so that a disc of accurate and uniform thickness is produced. Great care must be paid to haemostasis in the recipient cornea, and bleeding vessels should be cauterized if necessary. When complete haemostasis has been achieved, the graft is placed in position and held by indirect mattress sutures. I consider that interstitial and superficial vascularization of the graft is more likely to occur if direct sutures are used.

In the successful case, the graft remains relatively clear and free from vascularization, which, if present, is confined to the under surface of the graft between the graft and the recipient cornea.

After a variable period, sometimes up to 6 or 9 months, sensitivity develops in the graft and annular grafting may then be performed. The cornea is centred by the method of free-hand marking already described and a 10-mm. trephine cuts the cornea to a depth of $0.5 \mathrm{~mm}$. A concentric ring is now cut in the cornea with a 7-mm. trephine, again to a depth of $0.5 \mathrm{~mm}$. The annular strip of corneal tissue lying between these concentric cuts is dissected from the cornea, first by the use of the Paufique elevator and then with the Desmarres knife (Fig. 3).

By a similar method an annulus of the same dimensions is cut from the donor

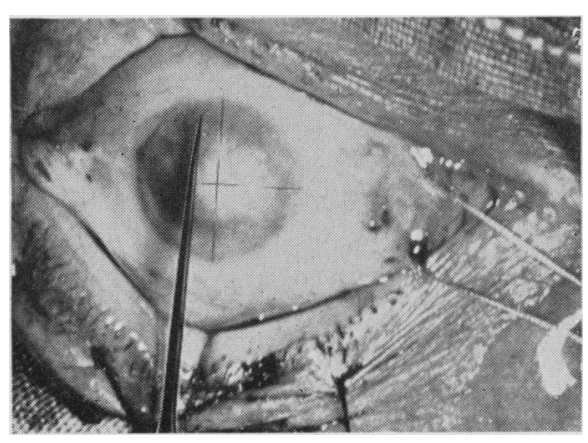

Fig. 2.-Marking cornea to obtain centration.

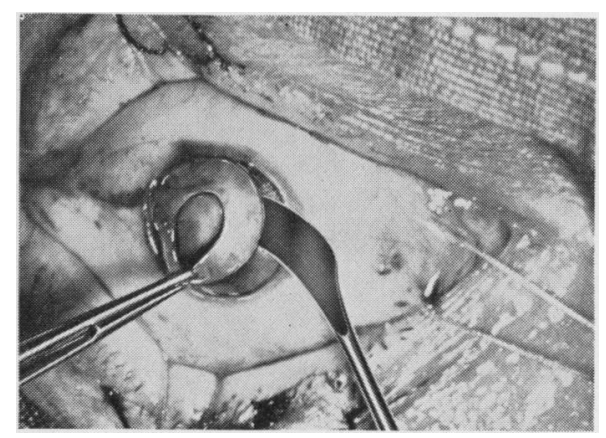

FIG. 3.-Disscction of annulus after concentric trephine cuts have been made. 
eye and is sewn into position (Figs 4 and 5) on the recipient cornea after haemostasis has been achieved. By the method of free-hand marking and cutting with the trephines, some slight eccentricity of the annulus sometimes results; when this occurs it is frequently seen in both donor and recipient cornea to a similar degree, and with suitable manipulation a perfect fit can be obtained.

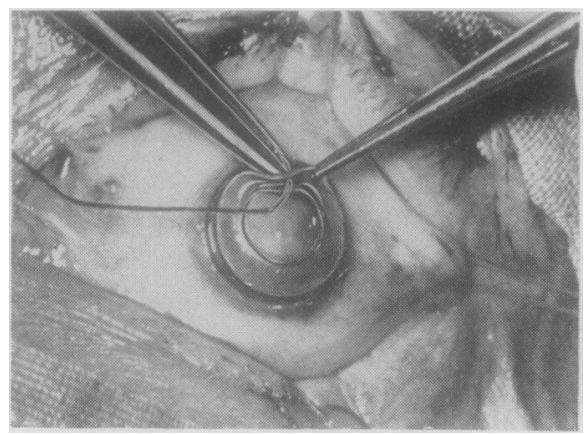

FIG. 4.--Suturing donor annulus into position.

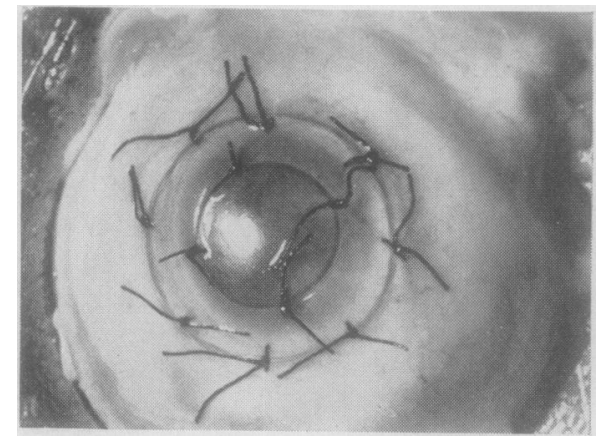

FIG. 5.-Appearance when suturing is completed.

The annular graft usually remains clear; some slight discrete, diffuse opacities are sometines seen and later sensitivity develops, the surrounding cornea clears, vascularization decreases, and even the opacity in the underlying cornea may become less dense.

When the sensitivity of the cornea has completely returned and if the grafts are clear or contain only discrete scattered opacities, then a final $5-\mathrm{mm}$. perforating graft may be performed. It cannot be emphasized too strongly that the final perforating graft must not be attempted unless both the central lamellar and the annular grafts are relatively clear, and for this reason I wait at least 6 months before proceeding to the next stage. If opacifications occur in either or both of the grafts, I repeat the grafts modifying the size and position as befits the individual.

\section{Case Reports}

Case 1, a man aged 46, had gross corneal opacificatiogn afte: repeated attacks of corneal ulceration over a period of several years. A dense marble-like opacity of the cornea extended through the whole thickness of an area some $7.8 \mathrm{~mm}$., the periphery of the cornea was clear, and vascularization slight (Fig. 6). A 7-mm. lamellar graft was placed centrally and little vascularization occurred. It was considered that an annular graft was not required owing to the clarity of the peripheral cornea and the small amount of vascularization which had developed-a 5-mm. perforating keratoplasty was therefore performed. This final operation was performed in July, 1953, and the graft is still absolutely clear (Fig. 7) giving a visual acuity of $6 / 6$.

Case 2, a man aged 37, had suffered from recurrent attacks of corneal inflammation for 10 years as a result of which a gross corneal opacity developed, the site of degenerative changes and superficial vascularization (Fig. 8).

After preliminary lamellar grafting (Fig. 9), gross vascularization developed between the graft and the recipient cornea. An annular graft was done a year later (October, 1953: Fig. 10) with considerable improvement in the appearance of the cornea. A year later a perforating keratoplasty (Fig. 11) was performed and this graft has now been clear for 9 months with a visual acuity of $6 / 9$. 


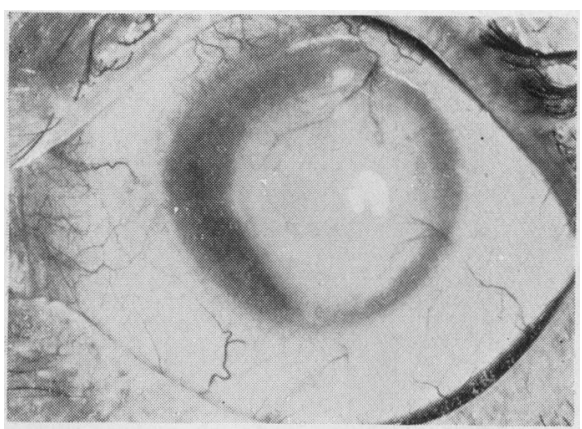

FIG. 6.-Case 1, before placing final perforating graft.

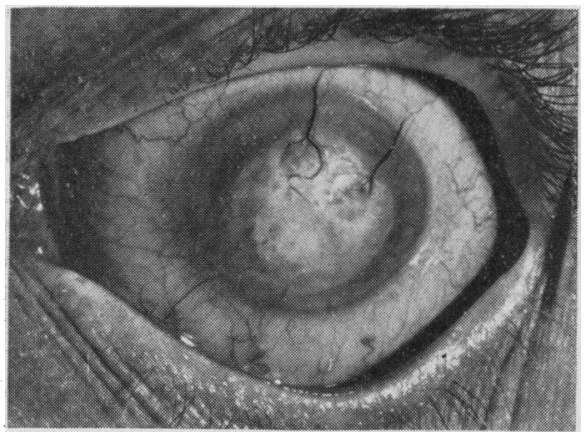

FIG. 8.-Case 2, pre-operative condition.

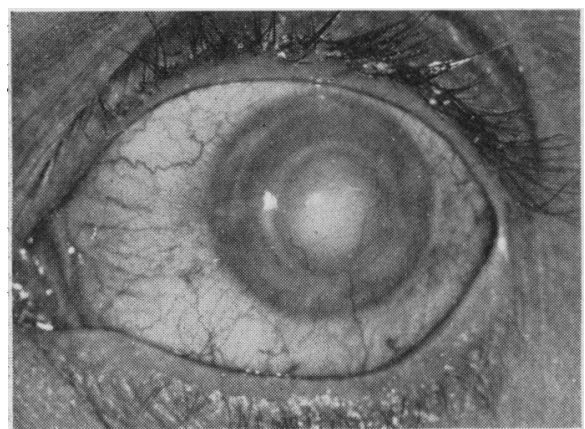

FIG. 10.-Case 2, after annular graft.

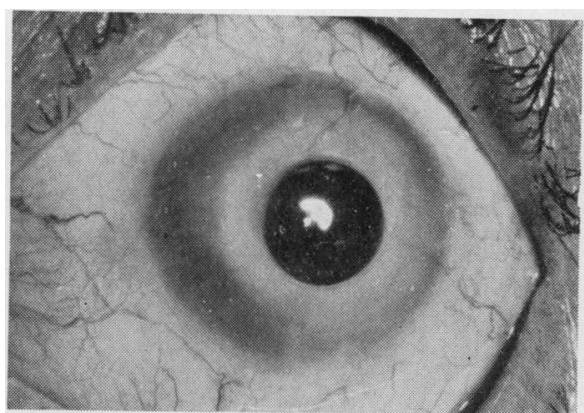

FIG. 7.-Case 1, after perforating graft.

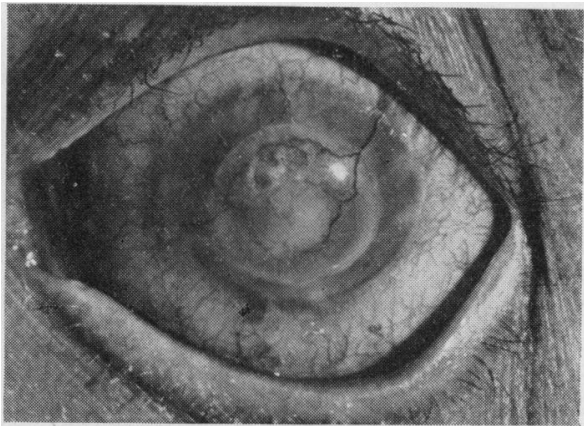

FIG. 9.-Case 2, after 6-mm. lamellar graft.

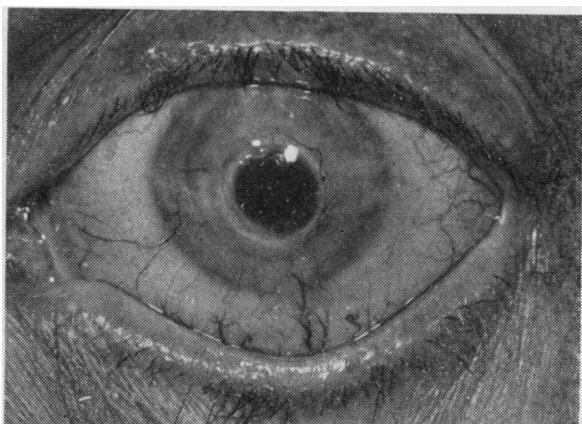

FIG. 11.-Case 2, after perforating graft.

Case 3, a man aged 65, had been gassed in 1917. The left eye showed a typical mustard gas keratitis but with the aid of a contact lens he had managed for many years with a visual acuity of 6/24. Recently the varicosities of the intra-corneal vessels had from time to time ruptured, causing an incapacitating intra-corneal haemorrhage which effectively obliterated his vision. His right eye had been quite blind ever since the gassing although light projection was accurate. The cornea of this eye was the seat of a gross opacity involving the entire thickness of the cornea with scattered areas of lipoid degeneration and observation subsequently confirmed by microscopy 


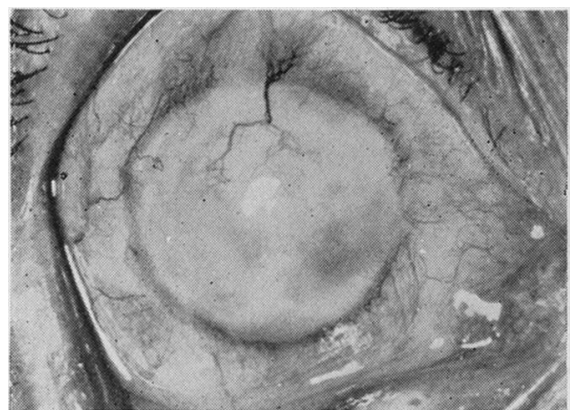

Fig. 12.-Case 3, after 6-mm. central lamellar graft.

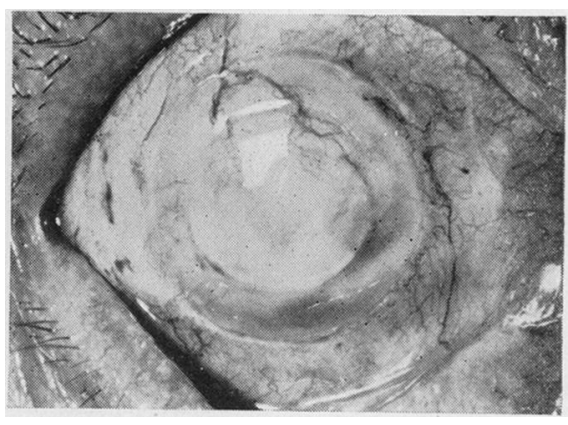

Fig. 13.-Case 3, after annular graft.

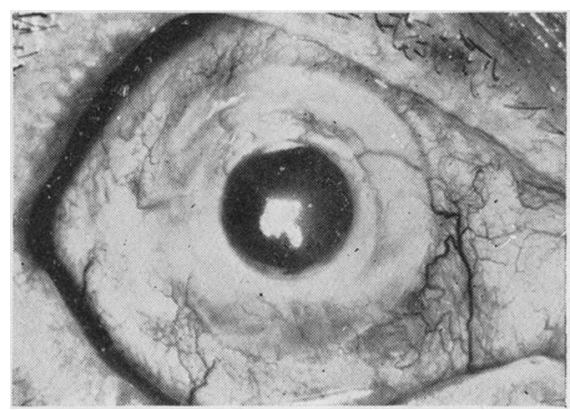

Fig. 14.-Case 3, after perforating graft.

I performed a lamellar graft on the right eye in January, 1953 (Fig. 12), an annular graft in June, 1953 (Fig. 13), and a perforating keratoplasty in January, 1954 (Fig. 14). Since that time the graft has remained absolutely clear, and the patient's corrected vision is $6 / 12$ and he can easily read N. 5.

I wish to express my indebtedness to Dr. Peter Hansell and the staff of the Department of Medical Illustration at the Institute of Ophthalmology and to Dr. P. N. Cardew of the Photographic Department of St. Mary's Hospital for the illustrations. 